\title{
Fully Polarimetric Microwave Radiometer for Remote Sensing
}

\author{
Janne Lahtinen, Student Member, IEEE, Jörgen Pihlflyckt, Ilkka Mononen, Simo J. Tauriainen, Martti Kemppinen, \\ and Martti T. Hallikainen, Fellow, IEEE
}

\begin{abstract}
The design, characteristics, and operation of the Helsinki University of Technology Fully Polarimetric Radiometer (FPoR) are described. The developed 36.5-GHz radiometer can be used for airborne remote sensing; however, ground-based and laboratory measurements are also possible. A direct cross-correlation technique with analog correlators, which measures all four Stokes parameters simultaneously, is applied. This paper is the first successful demonstration of an analog direct cross-correlation technique for polarimetric remote sensing radiometry. The radiometer was subjected to a variety of laboratory tests, and considerable attention is given to analysis of the characteristics of the instrument. Owing to the effective active temperature control system of the receiver, the radiometric stability of the instrument was found to be very high; test results showing stabilities below $10 \mathrm{mK}$ and of 4-40 mK on time scales of 800 and $8000 \mathrm{~s}$, respectively, are presented. Furthermore, the absolute accuracy of the system is analyzed to be at a sub-Kelvin level for most measurement conditions. A maritime wind vector experiment was carried out over the Gulf of Finland. The feasibility and performance of the applied correlation technique and the whole radiometer system were verified for fully polarimetric airborne measurements. The obtained brightness temperatures of the first three Stokes parameters show typical harmonic behavior with respect to the surface wind; the results suggest, however, that the model coefficients presented earlier for oceans may not be directly applicable for different conditions.
\end{abstract}

Index Terms-Microwave technology, polarimetry, radiometers, wind.

\section{INTRODUCTION}

$\mathbf{M}$ ICROWAVE radiometry using vertical and horizontal polarizations is a well-established technique for various remote sensing applications [1]. In radio astronomy, supplementary information has been obtained since the 1950 s by mea-

Manuscript received August 8, 2002; revised March 11, 2003. This work was supported in part by the National Technology Agency of Finland under Contracts 4276/96, 40266/97, and 40206/98, by the Graduate School in Electronics, Telecommunication and Automation (GETA), by the Jenny and Antti Wihuri Foundation, by the Vilho, Yrjö and Kalle Väisälä Foundation, by the Foundation of Technology, and by The Foundation for Commercial and Technical Sciences (KAUTE).

J. Lahtinen was with the Laboratory of Space Technology, Helsinki University of Technology. He is now with the European Space Agency, ESTEC, TOS-ETP, NL-2200 AG Noordwijk ZH, The Netherlands (e-mail: janne.lahtinen@esa.int).

J. Pihlflyckt, I. Mononen, S. J. Tauriainen, and M. T. Hallikainen are with the Laboratory of Space Technology, Helsinki University of Technology, FIN-02015 HUT, Espoo, Finland (e-mail: rap@avasun.hut.fi; mononen@avasun.hut.fi; simo@avasun.hut.fi; mhallika@avasun.hut.fi).

M. Kemppinen was with the Laboratory of Space Technology, Helsinki University of Technology. He is now with the Mikkeli Polytechnic Research Center YTI, FIN-50101 Mikkeli, Finland (e-mail: martti.kemppinen@mikkeliamk.fi).

Digital Object Identifier 10.1109/TGRS.2003.813847 suring the polarization characteristics of microwave emissions [2]. During the last decade, there has also been a growing interest in passive polarimetric remote sensing measurements. In particular, there have been several studies into the application of polarimetric radiometry for the retrieval of maritime wind vectors (e.g., see [3]-[6]).

Radiation fields are characterized in full using Stokes parameters. In remote sensing radiometry, it is convenient to use the modified Stokes parameters [7] in brightness temperature: the first $\left(T_{v}\right)$ and second $\left(T_{h}\right)$ parameter describe, respectively, the vertically and horizontally polarized brightness temperatures, whereas the third $\left(T_{3}\right)$ and fourth $\left(T_{4}\right)$ parameter describe, respectively, linearly and circularly polarized components. For simplicity, the term Stokes parameters is applied in the following for modified Stokes parameters in brightness temperature.

The measurement of the first three Stokes parameters enables the determination of surface wind speed and direction in sea areas [5], [6], which is valuable for many meteorological and oceanographic applications. Furthermore, $T_{3}$ also is sensitive to the orientation distribution of hydrometeors [8]. The measurement of $T_{4}$, on the other hand, is potentially valuable not only for vertical sounding of the mesosphere [9] but also for the retrieval of maritime wind vectors [4], [6]. For spaceborne polarimetric measurements $T_{4}$ would have an additional benefit of being unaffected by Faraday rotation. Over the last few years, the foreseen applications of polarimetric radiometry have inspired the development of several tripolarimetric [3], [10], [11] and fully polarimetric airborne radiometers [12]-[14], which measure, respectively, three or all four Stokes parameters at a time. The first spaceborne polarimetric radiometer is the U.S. Air Force's WindSat onboard the Coriolis satellite [15]. The satellite was launched in January 2003.

Helsinki University of Technology (HUT), Laboratory of Space Technology has developed a multichannel airborne radiometer system HUTRAD [16]. The ever-increasing interest in polarimetric microwave radiometry provided the motivation for also including a profiling polarimetric radiometer in the system: the fully polarimetric radiometer (FPoR).

FPoR was introduced in [12]; this paper includes a detailed description of the device. The theoretical background of polarimetric microwave radiometry and the requirements set for the FPoR are discussed in Section II. The instrument is described in Section III, and the characteristics are presented and analyzed in Section IV. The use of the FPoR for the measurement of wind-generated brightness temperature scenes is demonstrated in Section V. Conclusions are presented in Section VI. 


\section{THEORETICAL BACKGROUND AND REQUIREMENTS}

In developing FPoR and the whole HUTRAD system, the functional parameters were selected to guarantee a high compatibility with existing and future satellite instruments. Adopting the specifications presented in [17] for a $36.5-\mathrm{GHz}$ receiver, the requirements for radiometric resolution, stability, and accuracy of the FPoR were set to $0.5,0.5$, and $1.5 \mathrm{~K}$, respectively. In this resolution figure, 1 -s integration time and $250-\mathrm{K}$ antenna temperature are assumed.

A total power radiometer provides the best theoretical radiometric resolution of different radiometer topologies; the theoretical resolution of Dicke-switching and noise injection radiometers is degraded by a factor of two [18]. In practice, however, a total power radiometer requires frequent calibration to suppress the offset variations and gain variations of the receiver. The Dicke topology, on the other hand, cancels receiver noise variations and greatly reduces gain fluctuations, and the calibration interval can thus be considerably longer. After considering the technical complexity and achievable radiometric resolution and stability of different radiometer topologies, the Dicke-switching technique was selected for the FPoR. An option to measure in total power mode was also included. Note that although the fluctuations of system gain $\left(\Delta G / G_{0}\right)$ are reduced using the Dicke-switching topology, they are not completely cancelled [1, p. 372]. Since many RF components have nonnegligible temperature coefficients associated with them, effective temperature stabilization was set as a design goal to minimize $\Delta G / G_{0}$.

With regard to airborne and spaceborne remote sensing applications, there are several potential polarimetric receiver architectures. Considering the trade-offs associated with the different techniques, none of these techniques has so far proven to be superior. In view of receiver complexity, performance, and cost, however, digital direct cross correlation [11] and analog direct cross-correlation techniques are lucrative options in realizing a polarimetric receiver. These techniques estimate $T_{3}$ and $T_{4}$, respectively, by direct cross correlation of $T_{v}$ and $T_{h}$ in phase and quadrature (e.g., see [11] or [19]). After considering the trade-offs of different techniques, direct correlating analog topology was deemed to be the most suitable option for our purposes. The main advantages of the direct correlating analog correlators over digital ones include up to 1.57 times improved radiometric resolution of the polarimetric channels (i.e., the channels measuring $T_{3}$ and $T_{4}$ ) [20] and the simplicity of the device [21]. Furthermore, the power consumption is low. It is noted that the radiometric resolution of a digital correlator can be improved by oversampling and/or increasing the number of quantization levels [20]. These methods, however, may increase the complexity of the device.

The radiometric resolution of the polarimetric channels can be determined by applying the SNR of a single baseline in an aperture synthesis radiometer. The resolution is degraded due to differences of signal propagation times through the two channels to be correlated, as well as the differences in the transfer functions of these channels [22]. This effect is, in fact, a zerobaseline special case of the fringe-washing function, which is well known from the theory of interferometry [23]. Assuming

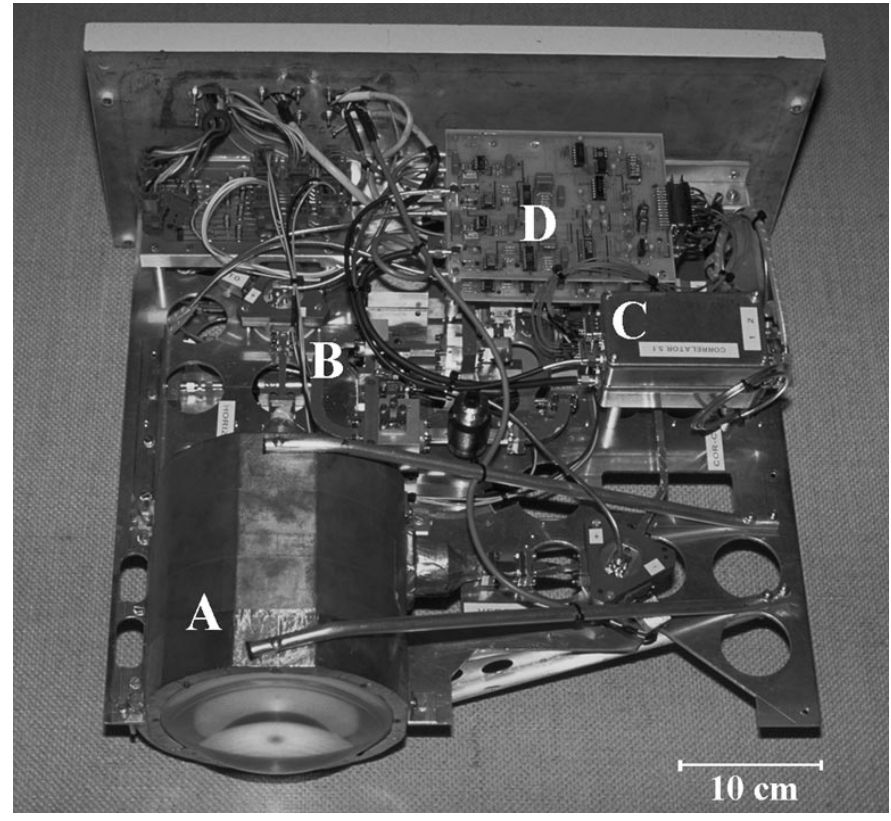

Fig. 1. Exposed view of the fully polarimetric radiometer receiver. (A) Lens loaded horn antenna, (B) radio frequency system on the upper instrumentation deck, (C) analog correlator units, and (D) low-frequency electronics system. The intermediate frequency system and a part of the correlator system are attached on the lower instrumentation deck and cannot be seen here.

an analog direct correlating Dicke-switching radiometer and applying [21]-[23], the radiometric resolutions of the $T_{3}$ and $T_{4}$ can be written as

$$
\begin{aligned}
\Delta T_{3 / 4} & \approx \frac{2}{D_{3 / 4}} \cdot \sqrt{\frac{2 \cdot T_{\mathrm{SYS}, v} T_{\mathrm{SYS}, h}}{\beta \cdot \tau}} \\
& =2 \cdot \sqrt{\frac{2 \cdot T_{\mathrm{SYS}, v} T_{\mathrm{SYS}, h}}{B_{3 / 4} \cdot \tau}} \\
B_{3 / 4} & =\frac{\left|\int_{0}^{\infty} H_{v} H_{h}^{*} d f\right|^{2}}{\int_{0}^{\infty}\left|H_{v}\right|^{2}\left|H_{h}^{*}\right|^{2} d f}
\end{aligned}
$$

where $T_{\mathrm{SYS}, v}$ and $T_{\mathrm{SYS}, h}$ are the system noise temperatures of the vertical and horizontal channels, respectively; $D_{3 / 4}$ is the degradation factor generated by the amplitude and phase variations of transfer functions; $\beta$ is the passband bandwidth; $B_{3 / 4}$ is the predetection bandwidth; and $H_{v}$ and $H_{h}$ are the complex voltage transfer functions of the vertical and horizontal channels, respectively, prior to correlation. The asterisk denotes a complex conjugate. It is assumed that the propagation time difference of the two signals is very small compared to the correlation time of the channels $(\sim 1 / B)$. It is further presumed that the polarized brightness temperature components of the scene are small, which is a reasonable assumption for most remote sensing targets. Note that if the phase differences of the transfer functions are small prior to correlation and the gain fluctuations $\left(\Delta G / G_{0}\right)$ of the orthogonal channels (i.e., the channels measuring $T_{v}$ and $T_{h}$ ) are small, (1) becomes $\Delta T_{3 / 4} \approx$ $\left(2 \cdot \Delta T_{v} \cdot \Delta T_{h}\right)^{1 / 2}$. The $\Delta T_{v}$ and $\Delta T_{h}$ are the radiometric resolutions of $T_{v}$ and $T_{h}$, respectively; for the well-known definition, see for example [1] or [18]. Because only correlated signals give an output, the gain variations will not affect the radiometric 


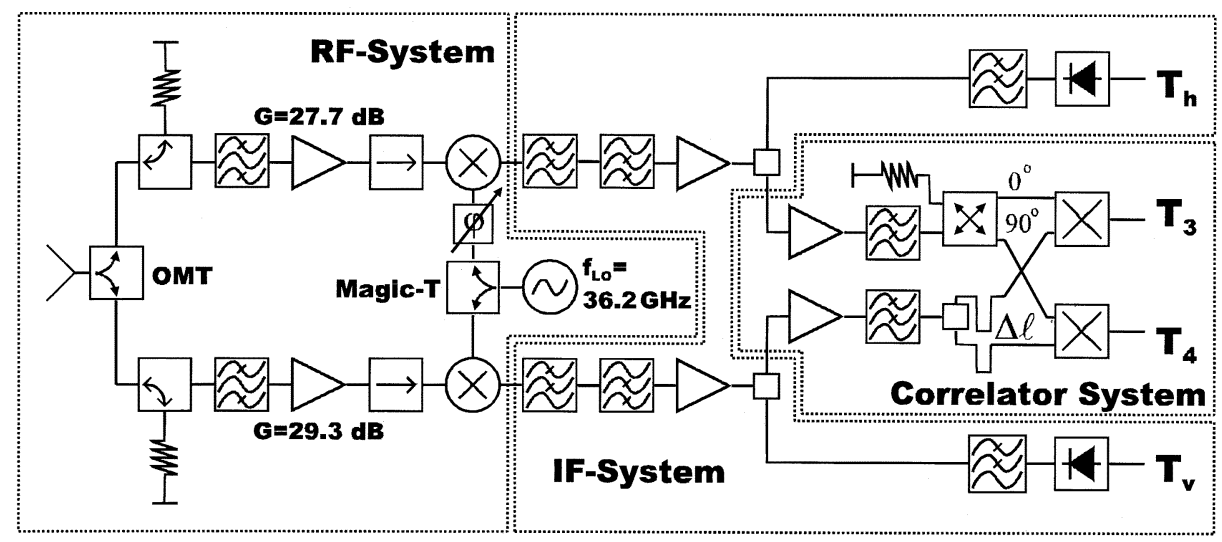

Fig. 2. Block diagram of the fully polarimetric radiometer receiver (excluding the LF electronics system).

resolution of the polarimetric channels, given that the correlated signals are low compared to system noise temperatures and the gain fluctuations are not correlated, e.g., due temperature drift.

In order to detect $T_{4}$, the orthogonal signals have to be correlated in quadrature. With regard to a superheterodyne receiver, the $90^{\circ}$ phase shift can be generated either in the RFor IF-line of the receiver. The latter solution was selected for the FPoR, since the RF-solution is associated with the increase of system complexity. The IF-solution, however, requires single side-band (SSB) receiver topology, as the upper and lower sidebands would otherwise cancel each other out upon the detection of $T_{4}$.

To accurately retrieve certain geophysical parameters, e.g., maritime wind vectors, stringent requirements are set for the absolute accuracy of polarimetric radiometer measurements [19]. In designing the FPoR, the following error sources were considered. Calibration uncertainties including the uncertainties in determining the crosstalk (i.e., signal leakage) between distinct channels [24], the errors in compensating for the motion of the aircraft, the receiver instabilities, and the side lobe level of antenna. Accurate compensation for the motion of the aircraft is crucial when retrieving the real brightness temperatures of the scene; the rotation of instrument polarization basis with respect to the polarized target can lead to considerable mixing of the first three (but not the fourth) Stokes parameters [7]. Furthermore, the variations of incidence angle can also have a significant impact on the brightness temperature of the orthogonal polarizations [1]. The development of an effective temperature stabilization system for the radiometer receiver was identified as very important; the fluctuations of the physical temperature deteriorate the accuracy by generating long time gain fluctuations and variations of resistive and reflective losses in the receiver front-end. As discussed above, the temperature fluctuations also generate short-term gain variations, which degrade the radiometric resolution.

\section{INSTRUMENT DESCRIPTION}

\section{A. General Description}

The developed FPoR operates at $36.5 \mathrm{GHz}$, and it is capable of simultaneous measurement of all four (modified) Stokes parameters. The radiometer can be operated either in total power or in Dicke-switching mode; for reasons of better stability, however, the Dicke-switching mode is usually preferred. The FPoR is integrated into the profiling subsystem of the HUTRAD radiometer system [16], which consists of several major assemblies networked together to form a local area network: The High Frequency Sensor Unit (HFSU) and its Power and Control Unit (PCU), the Low Frequency Sensor Unit (LFSU), and the Operator Interface Assembly (OIA). Although the HUTRAD is designed primarily for airborne measurements, laboratory and other ground-based measurements are possible as well. For airborne measurements, the OIA is mounted into the cabin of Short SC7 Skyvan research aircraft, whereas the other assemblies are mounted into the rear cargo bay. During a flight, the antenna incidence angle can be adjusted by altering the pitch angle of the aircraft; using nominal level flight parameters, the incidence angle is approximately $48^{\circ}$ to nadir. An end-to-end calibration of the radiometer from antenna to $\mathrm{A} / \mathrm{D}$ converters is performed prior to and after a measurement (flight) using conventional blackbody targets and the Fully Polarimetric Calibration Standard [24], [25], a passive standard that generates adjustable fully polarimetric brightness temperature scenes. Details of the individual subsystems of the FPoR and the HUTRAD are presented in the following.

\section{B. Microwave Receiver}

The FPoR receiver is installed into the housing of HUTRAD's High Frequency Sensor Unit. Functionally, the receiver consists of four systems: 1) radio frequency (RF) system, 2) intermediate frequency (IF) system, 3) low-frequency (LF) electronics system, and 4) correlator system. The components of the receiver are attached to a two-deck instrumentation tray. An exposed view of the receiver is shown in Fig. 1, the block diagram of the microwave circuit in Fig. 2, and the main functional parameters in Table I.

In the RF system, a Gaussian optics lens antenna splits the incoming microwave radiation into two parallel receiver chains for vertical and horizontal channels. These chains were made as identical as possible; for practical reasons, however, the vertical receiver chain includes an additional $90^{\circ}$ twist and somewhat longer waveguides, resulting in a $15-\mathrm{cm}$ difference in the electrical path length. Since the antenna is integrated into the instrumentation tray, antenna feeds are short and the feedline 
TABLE I

SySTEM CHARACTERISTICS OF THE FULLY POLARIMETRIC RADIOMETER OF THE HELSINKI UNIVERSITY OF TECHNOLOGY. a THE CHANNELS MEASURING $T_{v}, T_{h}, T_{3}$, AND $T_{4}$, RESPECTIVELY. $\mathrm{b}$ THE CHANNELS MEASURING $T_{3}$ AND $T_{4}$, RESPECTIVELY. ${ }^{\mathrm{C}}$ INCLUDES THE EFFECT OF THE ANTENNA. ${ }^{d}$ VERTICAL AND HORIZONTAL CHANNELS, RESPECTIVELY. ${ }^{e} 1$-s INTEGRATION TIME, 250-K ANTENNA TEMPERATURE. ${ }^{\mathrm{f}}$ OVER $800 \mathrm{~s}$ FOR THE ORTHOGONAL CHANNELS, OVER $8000 \mathrm{~s}$ FOR THE POLARIMETRIC Channels. ${ }^{\circ}$ During a SAmple Airborne Wind Vector Measurement, POTENTIAL NONLINEARITY NOT INCLUDED

\begin{tabular}{|c|c|}
\hline Parameter (Unit) & Value \\
\hline Center Frequency $(\mathrm{GHz})$ & 36.5 \\
\hline Intermediate Frequency (MHz) & $\sim 90-520$ \\
\hline Measured Polarizations & $T_{\mathrm{v}}, T_{\mathrm{h}}, T_{3}, T_{4}$ \\
\hline Antenna Type & Lens Loaded Horn Antenna \\
\hline Antenna Side Lobe Level (dB) & -25 (E-plane), -21 (H-plane) \\
\hline Polarization Isolation of the Antenna & $>40 \mathrm{~dB}$ \\
\hline Antenna 3 dB Beam Width (deg) & 4.0 \\
\hline Incidence Angle in Airborne Use (deg) & $\sim 40-60$ \\
\hline Integration Time (ms) & $50-3000$ \\
\hline Predetection Bandwidth $(\mathrm{MHz})^{a}$ & $430,415,417,414$ \\
\hline Degradation Factor $(\%)^{\mathrm{b}}$ & $98.0,98.1$ \\
\hline Receiver Noise Temperature $(\mathrm{K})^{\mathrm{c}, \mathrm{d}}$ & 1570,1210 \\
\hline Radiometric Resolution $(\mathrm{K})^{\mathrm{a}, \mathrm{e}}$ & $0.21,0.17,0.29,0.29$ \\
\hline$\Delta \mathrm{G} / \mathrm{G}^{\mathrm{d}}$ & $4.1 \times 10^{-3}, 4.8 \times 10^{-3}$ \\
\hline Stability $(\mathrm{mK})^{\mathrm{a}, \mathrm{f}}$ & $7,6,4,6$ \\
\hline Absolute Accuracy $(\mathrm{K})^{\mathrm{a}, \mathrm{g}}$ & $0.5,0.6,0.5$, and 0.8 \\
\hline
\end{tabular}

losses are thus minimized. Furthermore, the antenna and the transmission lines between the antenna and the Dicke-switches are enclosed in the same thermally stabilized enclosure as the rest of the receiver. Compared to systems where the temperature of the antenna(s) is not controlled, this solution improves both radiometric resolution and accuracy. The Dicke-switches are of a PIN-diode type with an insertion loss of $1.4 \mathrm{~dB}$; they are driven with a switching frequency of $1 \mathrm{kHz}$, which effectively suppresses the variations of receiver parameters.

As discussed in Section II, a single side-band (SSB) receiver topology was required. The spurious side-band signals are cancelled using RF-filters, which have a relatively high insertion loss $(0.8 \mathrm{~dB})$ due to the strict requirements set for image band rejection. The applied RF-amplifiers have a very wide bandwidth $(26-40 \mathrm{GHz})$; to protect the amplifiers from man-made radio frequency interference, the RF-filters are used as pre-selectors (i.e., in front of the RF-amplifiers). It is noted, however, that this configuration is not optimal with respect to receiver noise temperature; the noise that is generated in the image band by the filter-amplifier combination is amplified by the RF-amplifier and down-converted to the IF-band [26]. The noise figures for the amplifiers in the vertical and horizontal channels are approximately 2.2 and $2.3 \mathrm{~dB}$, respectively, at the applied frequency band.

Isolators were installed between the RF-amplifiers and mixers to suppress the possible leakage of the local oscillator signal out of the antenna, which could impair calibration if redirected back to the antenna [27]. We note that the isolators could also have been installed prior to the amplifiers; the current design, however, was chosen as the input matching of the mixers was considered to be more critical than that of the amplifiers. Furthermore, this choice is slightly better in terms of the overall noise temperature of the receiver. With regard to the overall absolute accuracy of the FPoR, the effect of the location of the isolators was simulated so as not to be unequivocal; the effect is, however, of the second order.

A direct correlating radiometer requires phase coherence between the orthogonal channels. Therefore, both receiver chains apply a common Gunn local oscillator (LO). An adjustable phase shifter in the LO-branch is used to level the phase characteristics of the orthogonal channels, which minimizes the crosstalk between the polarimetric channels; a phase unbalance causes both polarimetric channels to exhibit some sensitivity to $T_{3}$ and $T_{4}$. Note that signal leakage over the common local oscillator branch is a potential source of crosstalk between the orthogonal channels, and would also generate bias for the polarimetric signals [13]. To divide the LO-signal, we apply a magic-T hybrid, which has an isolation of over $20 \mathrm{~dB}$ between the colinear ports. Combined with the $20 \mathrm{~dB}$ RF-LO isolation of each mixer, the signal leakage is thus efficiently suppressed.

The IF system consist of filters, amplifiers, and square law detectors. Also, power dividers are included to provide the correlation system with the orthogonal signals. Due to the high stability over temperature, tunneling type detectors were selected to detect the vertical and horizontal signals.

The correlator system correlates the orthogonal signals in-phase and phase quadrature to measure $T_{3}$ and $T_{4}$, respectively. In essence, our system is constructed using two direct correlating analog correlator units [21] and a wide-band $90^{\circ}$ hybrid. The correlation system also provides additional amplification and filtering. In order to minimize the fringe-washing effect, the propagation time through vertical and horizontal chains was measured and delay lines were installed to balance the electrical lengths. Each correlator unit is built around a commercially available analog multiplier (Analog Devices AD 834) with only a few additional components. The topology is very straightforward and has several advantages, including small size, low weight, and cost effectiveness. The small number of components is also beneficial in terms of reliability. The applied multipliers exploit the Gilbert-cell structure with internal temperature compensation, which makes them insensitive to temperature fluctuations. Furthermore, the power consumption is only a few hundred milliwatts.

After the IF- and correlator systems, respectively, the detected orthogonal and polarimetric signals are fed to the LF electronics circuitry with some gain, Dicke demodulation, and digitizing. The radiometric signals are then transferred to the Power and Control Unit (PCU) and further to the Operator Interface Assembly (OIA).

\section{Power and Control Unit and Operator Interface Assembly}

The control electronics and voltage sources of the receiver are housed in a physically separate entity: the PCU. The PCU also accommodates the electronics for measuring the physical temperature of the receiver and an embedded computer to regulate the temperature. During calibration, the temperature sensors of the Fully Polarimetric Calibration Standard [24], [25] are connected to the PCU for temperature measurement.

The OIA incorporates the data archival system and the user interface. The radiometric and housekeeping data are recorded with a time stamp that is generated using a global positioning 
system (GPS) clock signal. This enables an accurate synchronization with the position and attitude data of the aircraft. A video camera is installed in the rear cargo bay of the aircraft and a video tape recording system is included in the OIA to support data interpretation.

\section{Temperature Control}

To suppress gain variations and to guarantee high measurement stability, close attention was paid to the temperature stabilization of the receiver. For optimum thermal stability, all components from the antenna to LF electronics are enclosed in a single temperature-stabilized enclosure.

The temperature of the receiver is stabilized to a user definable temperature by a proportional plus integral (PI) control routine. Conventionally, the temperature is set to $15 \mathrm{~K}$ above the expected ambient temperature during the measurement, which is near the natural thermal equilibrium of the system. The temperature is controlled using a thermo-electric Peltier-element that can both cool and heat a circulating airflow. The physical temperature of the receiver is monitored through four precision platinum resistive temperature detectors. As tested, the temperature control of the FPoR was found to be effective: after an initial warm-up period of $2 \mathrm{~h}$, the maximum peak-to-peak temperature fluctuations in laboratory conditions were $50 \mathrm{mK}$ over a time span of $25 \mathrm{~h}$.

\section{E. Attitude and Position Control}

Traditionally, airborne systems apply inertial navigational systems (INS) for attitude determination. Attitude-GPS systems, however, possess some advantages over INS, e.g., lower costs and absence of drifts over extended operation times [28]. Our measurement system incorporates both an attitude-GPS system [29] and an AISA spectrometer [30] GPS/INS-system, providing the position and the attitude of the aircraft (pitch, roll, and yaw). The inaccuracy of the attitude measurement is at a maximum of $0.1^{\circ}$.

\section{INSTRUMENT PERformance}

An extensive set of tests was carried out to characterize the performance of the Fully Polarimetric Radiometer; the obtained results are summarized in Table I.

The noise temperature of the orthogonal channels was measured using the standard $Y$-factor method. External calibration targets were applied, and thus the obtained values also include the antenna. It is noted that there is a nonnegligible difference between the noise temperatures. As the noise figures of the RF-amplifiers are well balanced, the result indicates $0.8 \mathrm{~dB}$ unbalance between the attenuations of the front-ends of the two channels; the estimated attenuations of vertical and horizontal channels are 3.2 and $2.4 \mathrm{~dB}$, respectively, prior to amplifiers. In general, the relatively high noise temperature is a consequence of using the image rejection filters as preselectors.

The radiometric resolution was determined as the standard deviation of the radiometer output when the antenna was illuminated using a stable blackbody target in ambient temperature. The same measurement and very long integration times also gave an estimate for the relative gain fluctuations $\left(\Delta G / G_{0}\right)$.
The results indicate that the measured radiometric resolution of the polarimetric channels is very close to $\left(2 \cdot \Delta T_{v} \cdot \Delta T_{h}\right)^{1 / 2}$; this suggests that the effect of the fringe-washing factor is small (see Section II). It is noted that although the system noise temperatures are increased by the selected architecture of the receiver front-end, the achieved radiometric resolutions are well within the design specifications.

Using a spectrum analyzer, the predetection bandwidths [18] of the orthogonal channels were estimated by measuring the gain variation over pass-bands. To study the characteristics of the polarimetric channels, a vector network analyzer (VNA) was applied to determine the complex transfer functions of the receiver chains prior to correlation. Note that due to technical limitations the IF sections and the RF sections were measured separately; the antenna and the mixers were not included in the measurement. As suggested by the measurement of radiometric resolution, the VNA measurements confirmed that the effect of the fringe-washing factor is small; first, the complex transfer functions of the channels are well balanced prior to correlator units as expressed by almost ideal degradation factors and second, the difference of the propagation times prior to correlation is negligible ( $0.06 \mathrm{~ns})$ compared to the correlation time (2.5 ns).

The antenna brightness temperature and the response vector of a fully polarimetric radiometer are connected via a four-element offset vector and a $4 \times 4$ element gain matrix, which also includes the interchannel crosstalk terms [19]. Using a fully polarimetric calibration standard, all unknown offset and gain terms can be solved to calibrate such a radiometer [19], [24]. In our case, however, it was possible to estimate by independent methods some of the calibration parameters that are substantially invariant over time; this a priori knowledge of the characteristics of the FPoR is used beneficially to improve calibration accuracy. Applying the Fully Polarimetric Calibration Standard [24], [25] as a reference target, brightness temperature measurements with a normal configuration were compared to the situation where the local oscillator branch of the horizontal channel was isolated. The results indicate a maximum polarization leakage level of $-30 \mathrm{~dB}$ between the orthogonal channels. Note that the possible cross polarization of the antenna is not included in this figure; according to the manufacturer, however, the polarization isolation of the antenna is better than $40 \mathrm{~dB}$, and the influence of the antenna can be ignored. Furthermore, the mixing of $T_{3}$ and $T_{4}$ into $T_{v}$ and $T_{h}$ was predicted to be $-30 \mathrm{~dB}$ at maximum, which is a reasonable assumption considering the layout of the receiver. The uncertainty of these estimates was set to $-30 \mathrm{~dB}$. Using the calibration procedure described in [24], the remaining unknown calibration coefficients of the FPoR have been determined in a variety of fully polarimetric calibrations both in the laboratory and in the field. The calibrations stretched over a period of one year. The calibration parameters were found to be dependent on the physical temperature of the receiver but relatively invariant on time, which could potentially be used beneficially to compensate any residual temperature fluctuations during measurements.

As a result of studying the mean calibration coefficient values obtained with different receiver temperatures, the following observations can be made. In general, the mixing of the orthog- 
onal signals into the polarimetric signals was found to be quite low: $-16 \mathrm{~dB}$ and $-20 \mathrm{~dB}$, respectively, from $T_{v}$ and $T_{h}$ into $T_{3}$ and $-21 \mathrm{~dB}$ and $-27 \mathrm{~dB}$, respectively, from $T_{v}$ and $T_{h}$ into $T_{4}$. Although the source of this mixing could not be identified, the most probable cause is the nonideality of the correlator units. The large variations in the crosstalk values can be explained by differences in component and subsystem level. The lowest coupling value $(-27 \mathrm{~dB})$ implies that crosstalk values close to those reported for digital correlators [11] are achievable by careful design and construction of a direct correlating analog cross correlator. Polarization mixing of $-14 \mathrm{~dB}$ was detected between the polarimetric channels, which indicates that the phases of the orthogonal channels have been balanced within a couple of degrees. Note that although the crosstalk terms are nonnegligible, they are compensated for in calibration.

The long-time stability of the FPoR was estimated in terms of Allan Standard Deviation (ASD), which is simply the square root of Allan variance [31]. A reference target was measured for $18 \mathrm{~h}$. With regard to the orthogonal channels, the ASD curve presents a typical "V" as a function of integration time, which indicates that white noise is the dominant noise type at short integration times, the amount of flicker noise is low, and the receiver instabilities become dominant at long integration times [32]. The corner for the white noise lies at around $800 \mathrm{~s}$, which corresponds to the longest integration time that improves the radiometric resolution; the stability of the $T_{v}$ and $T_{h}$ is approximately 7 and $8 \mathrm{mK}$, respectively, over $800 \mathrm{~s}$. The observed noise corner suggests an ideal calibration cycle of 10-15 min. However, the results also infer that the stability of the orthogonal channels remains at $20-40-\mathrm{mK}$ level on a time scale of $8000 \mathrm{~s}$. This indicates an insignificant drift over time periods of 2-3 h, which is a typical calibration cycle of an airborne measurement. Note that the observed target, a blackbody load in ambient temperature, was subjected to the temperature fluctuations of the laboratory. This hampered the interpretation of the results over long integration times, resulting in the high uncertainty in the ASD figure over $8000 \mathrm{~s}$. The polarimetric channels of the FPoR were found to be even more stable than the orthogonal channels; the results indicate a stability of $\sim 5 \mathrm{mK}$ over $8000 \mathrm{~s}$. To estimate the stability with different receiver physical temperatures, the dependence of the calibration parameters (gain and offset) on the physical temperature was applied. As simulated, the errors of the measured brightness temperatures are only weakly dependent on the mean receiver temperature by a certain amount of receiver temperature change. Assuming the temperature fluctuations to be the major cause for receiver instabilities, the simulation results infer that the measured stability values can be generalized for other receiver temperatures with reasonable accuracy.

In estimating the absolute accuracy of the FPoR in an airborne measurement, the following factors were considered. Calibration uncertainties as described in [24], the stability of the receiver and the dependence of the calibration parameters on receiver temperature, the side-lobe level of the antenna, and the inaccuracy of the attitude determination system. The radiometer was assumed to be linear. During calibration each calibration scene is observed for at least $30 \mathrm{~s}$; the radiometric resolution of such measurement is markedly below $0.1 \mathrm{~K}$, and the influence of the receiver noise on the absolute accuracy is effectively reduced. Note that the accuracy is dependent on several variables that vary between measurements, and no universal estimate of the accuracy can therefore be given. These variables include the brightness temperature vector of the scene, the calibration coefficients, which are linked to the physical temperature of the receiver, the stability of the receiver temperature, which can be degraded by large variations of ambient temperature, and the attitude of the aircraft. In general, however, the pixel-to-pixel absolute accuracy falls within the sub-Kelvin range given that the aircraft roll angle is below $\sim 25^{\circ}$, which is the case for most measurements. As a representative example, the accuracy has been estimated in Section V for a conducted wind vector measurement.

\section{Wind VECTOR MEASUREMENT}

To verify the airborne function of the FPoR and to study the dependence of polarimetric brightness temperatures on maritime wind vectors, a flight campaign was carried out in March 2002. The test area was 15-20 km off the coast near the Kallbådagrund weather station in the Gulf of Finland, the Baltic Sea. The Baltic Sea is a semienclosed brackish water basin in northern Europe. Circular flights collected brightness temperature signatures as a function of the azimuth angle relative to surface wind. By altering the roll and pitch angles of the aircraft between separate sets of circular flights it was possible to collect data with a variety of incidence angles. The Kallbådagrund weather station provided in situ data on several parameters, including wind speed and direction, atmospheric pressure, and temperature. The wind speed was $9.0 \mathrm{~ms}^{-1}$ referenced to $19.5 \mathrm{~m}$ above the surface. Using the data kindly provided by the Finnish Institute of Marine Research, the temperature and salinity of the water were estimated to be $+0.4{ }^{\circ} \mathrm{C}$ and $5 \mathrm{psu}$, respectively.

The fully polarimetric calibration of the raw data was performed as described in [24]. The attitude data of the aircraft was applied to compensate for the mixing of the first three Stokes parameters due to aircraft roll (banking) and the variations of the orthogonal signals due to incidence angle fluctuations. Atmospheric correction [1] was also applied.

A complete analysis of the results of the measurement campaign will be published elsewhere. A preliminary processing, however, has been carried out for seven datasets. Between these datasets, the roll and incidence angles of the antenna beam vary between $-36^{\circ} \cdots+39^{\circ}$ and $44^{\circ} \cdots 53^{\circ}$, respectively. An example of the retrieved Stokes parameters with respect to wind direction is presented in Fig. 3. In this particular dataset the incidence angle was $44^{\circ}$ and the banking of the aircraft ( $\sim$ the roll angle of the antenna beam) was $-2^{\circ}$. Each data point corresponds to approximately 3.9-s integration time and $100000 \mathrm{~m}^{2}$ of measured area. As can be seen, the potential harmonic modulation of the fourth Stokes parameter is very small $(<0.1 \mathrm{~K})$ and it could not be proven statistically. This result is in agreement with observations reported in [6] for $34 \mathrm{GHz}$. On the other hand, the orthogonal brightness temperatures and the third Stokes parameter demonstrate, respectively, even and odd harmonic azimuthal dependence on relative wind direction, as 

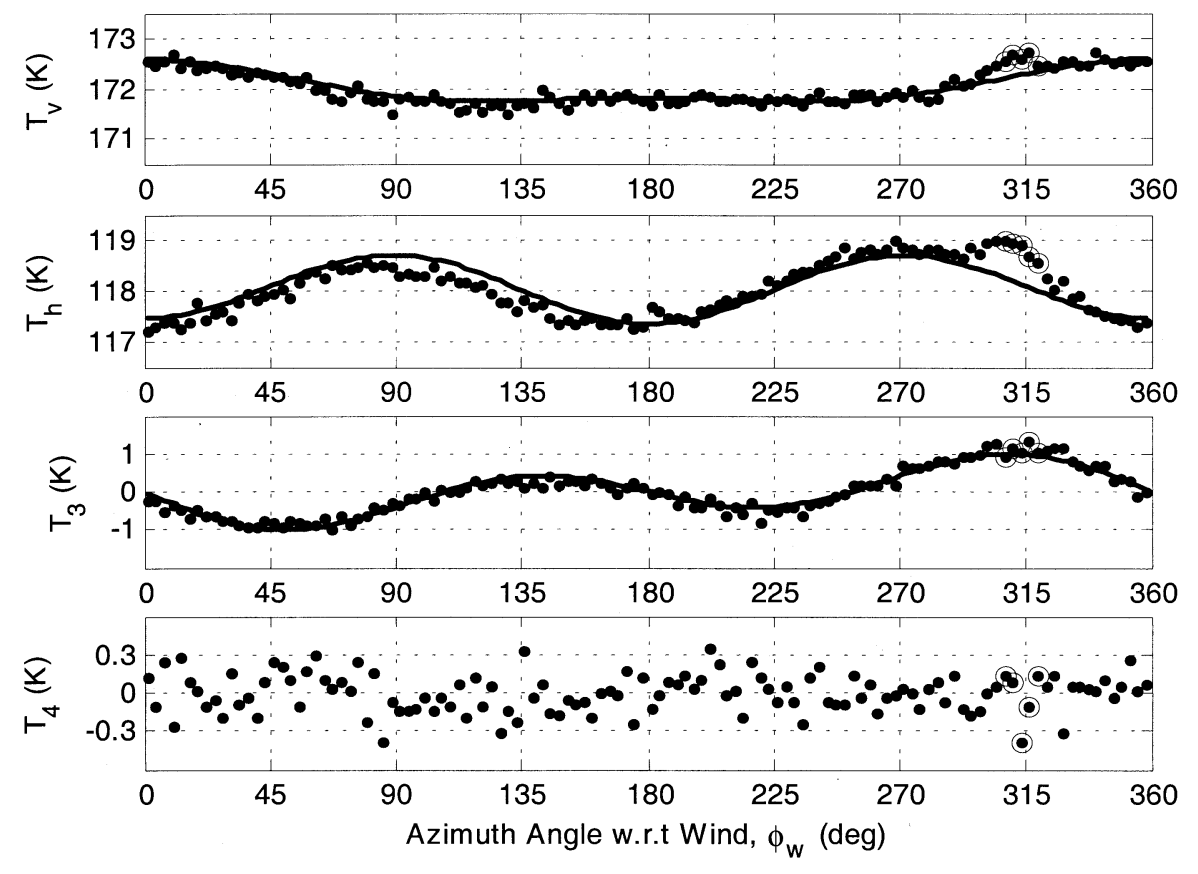

Fig. 3. Polarimetric brightness temperatures of the sea surface, measured using the FPoR. Individual points indicate the measurement values with respect to wind direction; the solid line represents the fitted second-order harmonic model for the first three Stokes parameters. Data points within $9^{\circ}$ of the direction of the sun are circled. The data were collected using an average incidence angle of $44^{\circ}$, and the in situ wind speed was $9.0 \mathrm{~ms}^{-1}$ referenced to $19.5 \mathrm{~m}$ above the surface.

TABLE II

HaRmonic COEFFicients of the Measured StOKes PARAMETERS. For COMPARISON, THE COEFFICIENTS BASED ON [4] ARE Given IN PARENTHESES

\begin{tabular}{llll}
\hline \hline & $T_{\mathrm{v}}(\mathrm{K})$ & $T_{\mathrm{h}}(\mathrm{K})$ & $T_{3}(\mathrm{~K})$ \\
\hline $1^{\text {st }}$ Harmonic & $0.38(1.0)$ & $0.05(0.1)$ & $-0.41(-0.8)$ \\
$2^{\text {nd }}$ Harmonic & $0.18(0.3)$ & $-0.65(-1.1)$ & $-0.69(-1.4)$ \\
\hline \hline
\end{tabular}

reported earlier, e.g., in [4]-[6]. Note that both orthogonal signals have a small protuberance due to the sun's reflection (sun glint) at around $310^{\circ}$ in azimuth; the data points within $9^{\circ}$ of the calculated mean azimuth of the sun are highlighted. Fitting of second-order harmonic models to the retrieved brightness temperatures gives very good results for the first three Stokes parameters. Excluding the corrupted data highlighted in the figure, the standard deviation from the model is $0.11,0.22$, and $0.15 \mathrm{~K}$ for $T_{v}, T_{h}$, and $T_{3}$, respectively. Compared with results reported earlier, these values suggest considerably lower-if not negligible-geophysical noise; for the retrieved data an instrumental noise of $0.11,0.09$, and $0.14 \mathrm{~K}$, respectively, was determined for $T_{v}, T_{h}$, and $T_{3}$. The standard deviation of $T_{4}$ was $0.14 \mathrm{~K}$, which is equal to the radiometric noise. The obtained first- and second-order harmonic coefficients of the first three Stokes parameters are presented in Table II; for comparison, the empirical model values presented in [4] for comparable wind speed, incidence angle, and frequency range are also included ( $T_{4}$ was not studied in [4] at this frequency range). Note that the coefficients that were obtained in this study are only about a half of those presented in [4]. This suggests that the relationship between the wind vector and the Stokes parameters may vary locally, as the measurements in [4] were carried out off the Californian coast in the Pacific
Ocean. Potential explanations include the absence of oceanic, long gravity waves in the test area and the differences in temperature and viscosity of the water, which have an influence on the foam/whitecap fraction [33]. The viscosity of the water, which is dependent on, for example, temperature, salinity, and impurities, also has a secondary effect on the generation of short waves [34]. Difference in atmospheric stratification is another potential explanation [35].

In estimating the absolute accuracy of this measurement, the error sources discussed in Section IV were accounted for. Assuming the different error sources to be independent, the overall pixel-to-pixel absolute accuracy becomes 0.5, 0.6, 0.5 , and $0.8 \mathrm{~K}$ for $T_{v}, T_{h}, T_{3}$, and $T_{4}$, respectively. The most significant error source is the calibration uncertainty. Note, however, that the absolute accuracy of the data was further enhanced assuming the third and fourth Stokes parameters to be zero mean over a full circle of azimuth [10].

Based on the preliminary analysis of the data, several observations can be made about the characteristics of the radiometer system. Due to high variations in the aircraft roll angle between the datasets, the coupling between the first three Stokes parameters varies considerably. The retrieved vertical and horizontal brightness temperatures, however, were found to be independent of the applied roll angle to within \pm 0.1 and $\pm 0.25 \mathrm{~K}$, respectively. The result indicates that the polarization leakage to the orthogonal channels of the FPoR is small, as has already been suggested in Section IV. Furthermore, the mean value of the retrieved $T_{3}$ and $T_{4}$ over a full circle of azimuth is close to zero for every flight configuration; the maximum deviation for $T_{3}$ and $T_{4}$ is, respectively, 0.3 and $0.7 \mathrm{~K}$. This suggests that the absolute accuracies of the polarimetric channels are within the estimated theoretical values. 


\section{CONCLUSION}

The Helsinki University of Technology Fully Polarimetric Radiometer (FPoR) has been designed and constructed for remote sensing at $36.5 \mathrm{GHz}$. The radiometer has been developed primarily for airborne applications, but it can also be applied for laboratory and ground-based (e.g., roof-top) measurements. The performance of the radiometer system has been tested in a variety of laboratory measurements. The requirements set for radiometric resolution, stability, and absolute accuracy were fulfilled. Especially noteworthy are the high stability of the receiver-particularly important for wind vector measurements [10] - and the precise calibration system [24], resulting in sub-Kelvin absolute radiometric accuracy for most measurement conditions. Note, however, that the influence of the possible nonlinearity of the device is not included in this figure. The feasibility of the radiometer for airborne measurements has been successfully verified in a wind vector experiment. The results indicate that FPoR is a useful tool in developing wind vector retrieval algorithms for present and future operational airborne and spaceborne satellite instruments (e.g., WindSat [15]). Other potential applications of the FPoR include, e.g., the detection of the distribution of hydrometeors.

The FPoR is based on analog direct cross-correlating topology. To the authors' knowledge this paper is the first demonstration of this technique for polarimetric remote sensing radiometry. The advantages of the applied solution include, for example, simple structure of both the receiver and the correlator, which reduces costs, increases reliability, and has low power consumption. The results demonstrate that the applied analog concept is a feasible and competitive option in building ground-based and airborne polarimetric radiometers. Furthermore, the results suggest that using a spaceborne polarimetric calibration standard proposed in [19] and [36], the analog technique is also a viable option for satellite instruments.

An airborne experiment was conducted over the Gulf of Finland; fully polarimetric brightness temperature signatures of the sea surface were obtained and compared to the azimuth angle of the surface wind. To the authors' knowledge this is the first presentation of polarimetric radiometer data that has been obtained of a semienclosed sea. The results of the fourth Stokes parameter suggest that the applicability of that parameter is very limited for wind vector determination at the applied incidence angle and frequency range. In line with earlier results (e.g., see [4] and [5]), however, the first three Stokes parameters show clear azimuthal modulation, confirming the usefulness of these parameters in retrieving surface wind vectors in sea areas. The geophysical noise of these parameters-especially of $T_{v}$ and $T_{3}$ - was found to be extremely small, indicating very homogeneous wave conditions in the test area. However, the harmonic coefficients of the azimuthal modulation were found to be only about half of those reported in [4]. This suggests that the relationship between the wind vector and the Stokes parameters may vary locally. The result emphasizes the importance of further study of the possible spatial variation of the polarimetric signature dependence on wind vectors; with regard to present and future operational airborne and spaceborne instruments, an improved accuracy in retrieving wind vector maps should prove beneficial for many local and global applications.

\section{ACKNOWLEDGMENT}

The authors wish to thank the contribution of A. Pyy, K. Rautiainen, E. Rinne, and P. Rummukainen in the measurements and in the development of the instrumentation. The help of M. Eskelinen and J. Praks with the attitude determination system in the airborne experiment is also highly appreciated. The support of K. Kahma and R. Hietala (Finnish Institute of Marine Research) is gratefully acknowledged. The authors also wish to acknowledge A. J. Gasiewski, J. Pulliainen, P. Piironen, and $\mathrm{O}$. Koistinen for their fruitful discussions.

\section{REFERENCES}

[1] F. T. Ulaby, R. K. Moore, and A. K. Fung, Microwave Remote Sensing: Active and Passive. Reading, MA: Addison-Wesley, 1981, vol. I.

[2] K. Akabane, "A polarimeter in the microwave region," Proc. IRE, pp. 194-197, Jan. 1958.

[3] M. S. Dzura, V. S. Etkin, A. S. Khrupin, M. N. Pospelov, and M. D. Raev, "Radiometers-polarimeters: Principles of design and application for sea surface microwave emission polarimetry," Proc. IGARSS, pp. 1432-1434, 1992.

[4] S. H. Yueh, W. J. Wilson, S. J. Dinardo, and F. K. Li, "Polarimetric microwave brightness signatures of ocean wind directions," IEEE Trans. Geosci. Remote Sensing, vol. 37, pp. 949-959, Mar. 1999.

[5] J. R. Piepmeier and A. J. Gasiewski, "High-resolution passive polarimetric microwave mapping of ocean surface wind vector fields," IEEE Trans. Geosci. Remote Sensing, vol. 39, pp. 606-622, Mar. 2001.

[6] B. Laursen and N. Skou, "Wind direction over ocean determined by an airborne, imaging, polarimetric radiometer system," IEEE Trans. Geosci. Remote Sensing, vol. 39, pp. 1547-1555, July 2001.

[7] L. Tsang, J. A. Kong, and R. T. Shin, Theory of Microwave Remote Sensing. New York: Wiley, 1985.

[8] A. Hornbostel, A. Schroth, A. Sobachkin, B. G. Kutuza, A. Evtuchenko, and G. K. Zagorin, "Passive and active stokes vector measurements of microwave emission and scattering by precipitation," Proc. IGARSS, pp. 2078-2080, 1999.

[9] P. W. Rosenkranz and D. H. Staelin, "Polarized thermal microwave emission from oxygen in the mesosphere," Radio Sci., vol. 23, pp. 721-729, Sept.-Oct. 1988.

[10] S. H. Yueh, W. J. Wilson, F. K. Li, S. V. Nghiem, and W. B. Ricketts, "Polarimetric measurements of sea surface brightness temperatures using an aircraft K-band radiometer," IEEE Trans. Geosci. Remote Sensing, vol. 33, pp. 85-92, Jan. 1995.

[11] J. R. Piepmeier and A. J. Gasiewski, "Digital correlation microwave polarimetry: Analysis and demonstration," IEEE Trans. Geosci. Remote Sensing, vol. 39, pp. 2392-2410, Nov. 2001.

[12] J. Lahtinen and M. Hallikainen, "A low-cost polarimetric radiometer for real time stokes parameter measurement," Proc. IGARSS, pp. 1597-1599, 1997.

[13] N. Skou, B. Laursen, and S. Sødjærg, "Polarimetric radiometer configurations: Potential accuracy and sensitivity," IEEE Trans. Geosci. Remote Sensing, vol. 37, pp. 2165-2171, Sept. 1999.

[14] I. Corbella, A. J. Gasiewski, M. Klein, V. Leuski, A. J. Francavilla, and J. R. Piepmeier, "On-board accurate calibration of dual-channel radiometers using internal and external references," IEEE Trans. Microwave Theory Tech., vol. 50, pp. 1816-1820, July 2002.

[15] P. W. Gaiser, "WindSat-Satellite-based polarimetric microwave radiometer," Proc. IEEE Microwave Symp. Digest, vol. 1, pp. 403-406, 1999.

[16] M. Hallikainen, M. Kemppinen, J. Pihlflyckt, I. Mononen, T. Auer, K. Rautiainen, and J. Lahtinen, "HUTRAD: Airborne multifrequency microwave radiometer," in Proc. ESA Workshop on Millimeter Wave Technology and Applications: Antennas, Circuits and Systems, 1998, pp. 115-120.

[17] E. Panula-Ontto, M. Hallikainen, M. Kemppinen, M. Nikulainen, J. Hyyppä, L. Kurvonen, A. K. Ghosh, and F. Zangerl, "Definition of Multi-channel Airborne Microwave Radiometer (MAMR)," ESA/ESTEC, Noordwijk, The Netherlands, 1995. 
[18] M. E. Tiuri, "Radio astronomy receivers," IEEE Trans. Antennas Propagat., vol. AP-12, pp. 931-938, Dec. 1964.

[19] J. Lahtinen, A. J. Gasiewski, M. Klein, and I. Corbella, "A calibration method for fully polarimetric microwave radiometers," IEEE Trans. Geosci. Remote Sensing, vol. 41, pp. 588-602, Mar. 2003.

[20] J. B. Hagen and D. T. Farley, "Digital-correlation techniques in radio science," Radio Sci., vol. 8, no. 8-9, pp. 775-784, Aug.-Sept. 1973

[21] O. Koistinen, J. Lahtinen, and M. Hallikainen, "Comparison of analog continuum correlators for remote sensing and radio astronomy," IEEE Trans. Instrumentat. Measurement, vol. 51, no. 2, pp. 227-234, Apr. 2002.

[22] A. R. Thompson and L. R. D'Addario, "Frequency response of a synthesis array: Performance limitations and design tolerances," Radio Sci. vol. 17, no. 2, pp. 357-369, Mar-Apr. 1982.

[23] A. R. Thompson, J. M. Moran, and G. W. Swenson, Jr., Interferometry and Synthesis in Radio Astronomy. New York: Wiley, 1986.

[24] J. Lahtinen and M. Hallikainen, "HUT fully polarimetric calibration standard for microwave radiometry," IEEE Trans. Geosci. Remote Sensing, vol. 41, pp. 603-611, Mar. 2003

[25] _ - "Fully polarimetric calibration system for HUT polarimetric radiometer," Proc. IGARSS, pp. 1542-1544, 2000.

[26] R. Pettai, Noise in Receiving Systems. New York: Wiley, 1984.

[27] D. M. Jackson, "Calibration of millimeter-wave radiometers with application to clear-air remote sensing of the atmosphere," Ph.D. thesis, Georgia Inst. Technol., Atlanta, GA, 1999.

[28] D. J. Dowgiallo, J. P. Bobak, N. R. McGlothlin, and K. M. St.Germain, "An economical and highly accurate attitude and position determination system for airborne polarimetric sensors," Proc. IGARSS, pp. $1620-1621,2001$.

[29] S. Tauriainen, J. Pihlflyckt, P. Ahola, and M. Hallikainen, "Multipurpose use of GPS on a flying remote sensing laboratory," Inst. Navigat. GPS-98, pp. 1223-1227, 1998

[30] J. Praks, "Operation of AISA spectrometer in FOREMMS airborne campaign," in Proc. URSI XXVI Convention on Radio Science and 2nd Finnish Wireless Communications Workshop, Digest of Technical Papers, M. Renfors, Ed: Tampere Univ. Technol., Inst. Commun. Eng., 2001, pp. 145-146

[31] D. W. Allan, "Statistics of atomic frequency standards," Proc. IEEE, vol 54, no. 2, pp. 221-230, Feb. 1966.

[32] G. Rau, R. Schieder, and B. Vowinkel, "Characterization and measurement of radiometric stability," in Proc. 14th European Microwave Conf., 1984, pp. 248-253

[33] I. V. Cherny and V. Y. Raizer, Passive Microwave Remote Sensing of Oceans. Bodmin, U.K.: Wiley, 1998.

[34] K. K. Kahma and M. A. Donelan, "A laboratory study of minimum wind speed for wind wave generation," J. Fluid Mech., vol. 192, pp. 339-364, 1988

[35] M. N. Pospelov, "Surface wind speed retrieval using passive microwave polarimetry: The dependence on atmospheric stability," IEEE Trans. Geosci. Remote Sensing, vol. 34, pp. 1166-1171, Sept. 1996.

[36] A. J. Gasiewski and D. B. Kunkee, "Calibration and application of polarization-correlating radiometers," IEEE Trans. Microwave Theory Tech. vol. 41, pp. 767-773, May 1993.

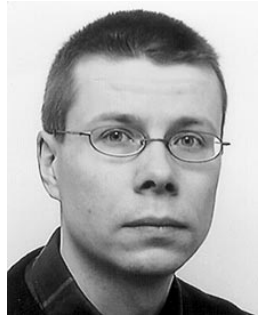

Janne Lahtinen (S'98) received the M.Sc. (Tech.) and Lic.Sci. (Tech.) degrees from the Helsinki University of Technology (HUT), Espoo, Finland, in 1996 and 2001, respectively.

He is currently a Research Fellow with the European Space Agency's European Space Research and Technology Centre (ESTEC), Noordwijk, The Netherlands. From 1995 to 2002, he was with the HUT Laboratory of Space Technology. His research has focused on microwave radiometer systems, with emphasis on polarimetric and interferometric radiometers. He has authored and coauthored 30 scientific publications focused on microwave remote sensing and other fields.

Mr. Lahtinen served as a Secretary of the Finnish National Committee of COSPAR from 1997 to 2002 and as a Secretary of the Space Science Committee, appointed by the Finnish Ministry of Education, from 1999 to 2000. He was awarded the third place in the IEEE Geoscience and Remote Sensing Society Student Prize Paper Competition in 2000, and in 2001 he won the Young Scientist Award of the Finnish National Convention on Radio Science.

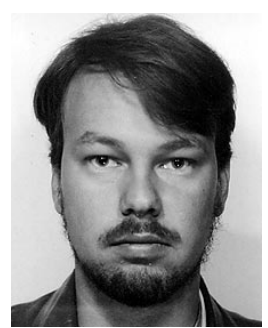

Jörgen Pihlflyckt is currently pursuing the M.S. degree at the Helsinki University Technology (HUT), Espoo, Finland.

He is currently a Research Associate in the Laboratory of Space Technology, HUT, which he joined in 1994. His current research interests include the development of electronics, software, and onboard local area network for the HUT airborne microwave radiometers.

Ilkka Mononen, photograph and biography not available at the time of publication.

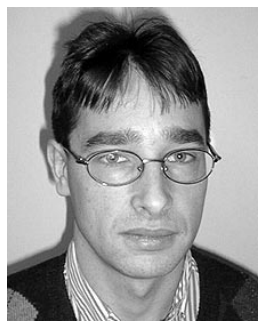

Simo J. Tauriainen received the M.Sc. (Tech) and Lic. Sci. (Tech) degrees from the faculty of Electrical Engineering, Helsinki University of Technology (HUT), Espoo, Finland, in 1993 and 1998 , respectively.

Since 1993, he has been a Research Scientist with the HUT Laboratory of Space Technology. His research interests include development of microwave remote sensing and navigation systems. He is an author and coauthor of 37 scientific publications.

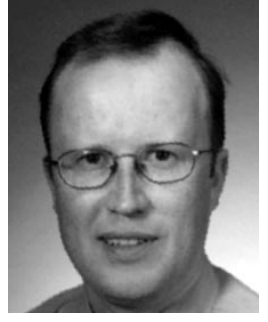

Martti Kemppinen received the M.Sc. (Tech.), Lic.Sci. (Tech.), and D.Sc. (Tech.) degrees from the Helsinki University Technology (HUT), Espoo, Finland, in 1989, 1991, and 1996, respectively.

He was with the Laboratory of Space Technology, Helsinki University Technology (HUT), Espoo, Finland, as a Research Scientist from 1988 to 1997 where his research interests included mechanical design of airborne remote sensing instruments, airborne platform modifications, and imaging microwave radiometry. He moved to industry in 1997, becoming an R\&D Engineer with a warehouse truck manufacturer Rocla Oyj, Järvenpää, Finland. In 1999, he became an R\&D Manager and later an R\&D Director at a mobile phone accessory manufacturer Moduleo Oy, Helsinki, Finland. Since 2001, he is the Research Director of the Mikkeli Polytechnic Research Centre YTI, Mikkeli, Finland, where he directs mechanical engineering and materials technology services to the industry. He is an author and coauthor of 40 scientific publications focused on the mechanical design of remote sensing instruments, and he has two patents concerning mobile phone accessories. Two of his publications have been internationally awarded. 


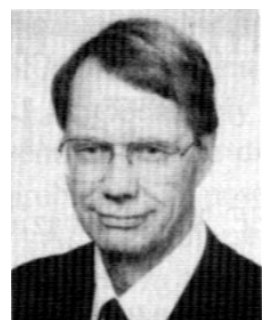

Martti T. Hallikainen (M'83-SM'85-F'93) received the M.S. degree in engineering and the Dir. Tech. degree from the Faculty of Electrical Engineering, Helsinki University of Technology (HUT), Espoo, Finland, in 1971 and 1980, respectively.

Since 1987, he has been a Professor of Space Technology at HUT, where his research interests include remote sensing and satellite technology. In 1988, he established the HUT Laboratory of Space Technology and serves as its Director. He was a Visiting Scientist from 1993 to 1994 at the European Union's Joint Research Centre, Institute for Remote Sensing Applications, Ispra, Italy. He was a Postdoctoral Fellow at the Remote Sensing Laboratory, University of Kansas, Lawrence, from 1981 to 1983, and was awarded an ASLA Fulbright Scholarship for graduate studies at the University of Texas, Austin, in 1974-1975. He is an author/coauthor of over 500 scientific publications.

Dr. Hallikainen served as President of IEEE Geoscience and Remote Sensing Society (IEEE GRSS) in 1996 and 1997, and as Vice President in 1994 and 1995. Since 1988, he has been a member of the IEEE GRSS Administrative Committee, and from 1999 to 2001, he served as the IEEE GRSS Nominations Committee Chair and since 2002, as the Fellow Search Committee Chair. He was the General Chairman of the IGARSS'91 Symposium and Guest Editor of the Special IGARSS' 91 Issue of the IEEE TRANSACTIONS ON GEOSCIENCE AND REMOTE SENSING (TGARS). Since 1992, he has been an Associate Editor of TGARS. He was a member of the IEEE Periodicals Committee in 1997 and Corresponding member of the IEEE New Technology Directions Committee from 1992 to 1995. He was Secretary General of the European Association of Remote Sensing Laboratories (EARSeL) from 1989 to 1993 and Chairman of the Organizing Committee for the EARSeL 1989 General Assembly and Symposium. He has been a member of the EARSeL Council since 1985, and he was a member of the Editorial Board of the EARSeL Advances in Remote Sensing from 1992 to 1993 . He has been a member of the European Space Agency's
(ESA) Earth Science Advisory Committee since 1998 and a member of the ESA SMOS Scientific Advisory Group since 2000. He was a national delegate to the ESA Earth Observation Scientific and Technical Advisory Group (EOSTAG) from 1988 to 1994, and he has served in the same capacity on the ESA Earth Observation Data Operations Scientific and Technical Advisory Group (DOSTAG) since 1995. He was Thematic Coordinator of the ESA EMAC-95 airborne campaign for Snow and Ice activities. He was a member of the ESA Multi-frequency Imaging Microwave Radiometer (MIMR) Expert Group from 1988 to 1994 and was a member of the ESA MIMR Scientific Advisory Group from 1994 to 1996. Since 1992, he has been a member of both the Advisory Committee for the European Microwave Signature Laboratory of the European Union's Joint Research Centre and the National Liaison of the International Space University. He is currently serving as Chair of Commission F International Union of Radio Science (URSI) from 2002 to 2005 and has served as its Vice Chair from 1999 to 2002. He was a member of the URSI Long Range Planning Committee from 1996 to 1999, a member of the URSI Committee on Geosphere and Biosphere Program from 1989 to 1999, and a URSI representative to SCOR from 1999 to 2002 . He has been a national official member of URSI Commission F (Wave Propagation and Remote Sensing) since 1988. He was Secretary of the Organizing Committee for the URSI Nordic Antenna Symposium in 1976, and he served as Secretary of the Finnish National Committee of URSI from 1975 to 1989. He was Vice Chair of the URSI Finnish National Committee from 1990 to 1996, and he has served as its Chair since 1997. He is Vice Chair of the Finnish National Committee of COSPAR since 2000. He is the recipient of three IEEE GRSS Awards: 1999 Distinguished Achievement Award, IGARSS'96 Interactive Paper Award, and 1994 Outstanding Service Award. He is the winner of the Microwave Prize for the best paper in the 1992 European Microwave Conference, and he received the HUT Foundation Award for excellence in research in 1990. He and his research team received the 1989 National Research Project of the Year Award from Tekniikka \& Talous (Technology \& Management Magazine). He received the 1984 Editorial Board Prize of Sähkö-Electricity in Finland. 